\title{
Efecto de la Temperatura y pH sobre la Actividad de las Enzimas Hidrolíicas de la Región Cecal de Cuyes (Cavia porcellus)
}

\author{
García, M. ${ }^{\circledR} ;$ Suarez, S. ${ }^{2}$; Carcelen F. ${ }^{1}$. and Olazabal, J. ${ }^{1}$
}

\begin{abstract}
'Laboratorio de Bioquímica, Nutrición y Alimentación Animal, Facultad de Medicina Veterinaria, Universidad Nacional Mayor de San Marcos, Lima. Perú.

${ }^{2}$ Centro de Investigación de Bioquímica y Nutrición, Facultad de Medicina Humana, Universidad Nacional Mayor de San Marcos, Lima. Perú.
\end{abstract}

\section{PalABras CLAVE ADICIONALES}

Hidrolasas.

Proteolítica.

Celulolítica.

Amilolítica.

Lipolítica.

\section{RESUMEN}

\begin{abstract}
El objetivo de este estudio fue evaluar el efecto de la temperatura y el $\mathrm{pH}$ sobre la actividad de las enzimas hidrolíicas de la región cecal de cuyes (Cavia porcellus). Este es el primer reporte sobre la actividad enzimática en el ciego de cuyes, evidenciando que existe actividad amilolítica, celulolítica, proteolítica y lipolítica. Los valores de las actividades evaluadas estuvieron en los siguiente rangos: actividad amilolítica (0.150 - $0.499 \mathrm{U} / \mathrm{mg}$ proteína), celulolítica (0.013 $-0.022 \mathrm{U} / \mathrm{mg}$ proteína), proteolítica (0.104 - $0.290 \mathrm{U} / \mathrm{mg}$ proteína) y lipolítica (2.227 - $4.049 \mathrm{U} / \mathrm{mg}$ proteína). Tanto en la actividad celulolítica, lipolítica y proteolítica hubo interacción entre temperatura y $\mathrm{pH}$. La actividad amilolítica solo se vio afectada por la temperatura, evidenciándose mayor actividad enzimática a mayor temperatura $\left(40^{\circ} \mathrm{C}\right)$. La actividad amilolítica es estable a cualquiera de los tres $\mathrm{pH}$ probados en este estudio. Además, la actividad amilolítica fue 10 veces más alta que la actividad celulolítica.
\end{abstract}

\section{Effect of Temperature and pH on the Activity of the Hydrolytic Enzymes of the Cecal Region of Guinea Pig (Cavia porcellus)}

\section{SUMMARY}

\section{ADDITIONAL KEYWORDS}

Hydrolases.

Proteolytic.

Cellulolytic.

Amylolytic.

Lipolytic.

\section{INFORMATION}

Cronología del artículo.

Recibido/Received: 08.02.2018

Aceptado/Accepted: 10.07.2019

On-line: 15.07.2019

Correspondencia a los autores/Contact e-mail:

madeline.garcia@unmsm.edu.pe
The aim of this study was to evaluate the effect of temperature and $\mathrm{pH}$ on the activity of hydrolytic enzymes in the cecal region of guinea pigs (Cavia porcellus). This is the first report on enzymatic activity in guinea pigs, showing that there is amilolytic, cellulolytic, proteolytic and lipolytic activity. The values of the evaluated activities were in the following ranges: amylolitic activity $(0.150-0.499 \mathrm{U} / \mathrm{mg}$ protein), cellulolytic (0.013 - $0.022 \mathrm{U} / \mathrm{mg}$ protein), proteolytic (0.104 - $0.290 \mathrm{U} / \mathrm{mg}$ protein) and lipolytic (2.227 - $4.049 \mathrm{U} / \mathrm{mg}$ protein). Both in the cellulolytic, lipolytic and proteolytic activity there was interaction between temperature and $\mathrm{pH}$. The amylolytic activity was only affected by the temperature, evidencing greater enzymatic activity at a higher temperature $\left(40^{\circ} \mathrm{C}\right)$. The amylolytic activity is stable at any of the three $\mathrm{pHs}$ tested in this study. In addition, the amylolytic activity was 10 times higher than the cellulolytic activity.

\section{INTRODUCCIÓN}

El cuy (Cavia porcellus) es un roedor oriundo de los Andes, utilizado como alimento en una extensa región comprendida por Bolivia, Perú, Ecuador y Colombia. Es considerado por la ONU y la FAO como una fuente de seguridad alimenticia para la población de escasos recursos económicos habitantes de estas zonas (Food and Agriculture Organization of the United Nations, 1997). Provee una carne exquisita y de alto valor nutricional. Según su fisiología digestiva, el cuy es un herbívoro de fermentación post gástrico. La mayor capacidad fermentativa en esta especie la realiza en el ciego (Esquerre, Valenzuela \& Candela, 1974), ana- 
tómicamente esta área representa el 35\% del sistema digestivo. En el ciego al igual que en las cámaras fermentativas de otros herbívoros está habitado por una microbiota muy numerosa y diversa, los cuales son los responsables de la utilización del alimento fibroso.

Esta microbiota posee una potente maquinaria enzimática que pueden romper los enlaces glucosídico $\beta$ 1-4 del alimento fibroso, ocurriendo así la digestión de alimentos fibrosos con la posterior producción y absorción de ácidos grasos volátiles de cadena corta, proteínas microbiales, vitaminas del complejo B y electrolitos. La microbiota del ciego se multiplica a un punto que sobrepasan a la acción de las enzimas proteolíticas, siendo menos eficiente el ciego en comparación al rumen. A pesar que el tiempo de multiplicación de la microbiota del ciego es mayor que la retención del alimento, los cuyes lo resuelven mediante mecanismos que aumentan la permanencia y en consecuencia la utilización de la digesta (Gomez \& Vergara, 1993).

Las enzimas se utilizan históricamente en procesos de fermentación como la fabricación de quesos, elaboración de vino, etc. (Lindal, 2004), actualmente sus aplicaciones industriales se proyectan además a la industria farmacéutica, cosmética y de alimentos, entre otros. El rápido desarrollo en la utilización de enzimas visto principalmente durante las últimas cinco décadas ha sido gracias a la evolución de la biotecnología moderna. Las fuentes de enzimas tradicionalmente han sido tejidos o microorganismos comestibles de animales y plantas que se utilizaron con seguridad en la elaboración de alimentos durante más de 1000 años. Con la creciente demanda de enzimas, se buscan nuevas fuentes de enzimas, principalmente de microorganismos silvestres (Buchholz, Kasche \& Bornscheuer, 2012); para trabajar estas nuevas fuentes de enzimas se requiere conocer las condiciones necesarias para su óptimo desarrollo, siendo la temperatura y el $\mathrm{pH}$ las condiciones básicas necesarias. Aún es desconocida la actividad enzimática hidrolítica y sus características en el ciego del cuy, así como su potencial importancia en el ámbito biotecnológico. Por las consideraciones expuestas y debido al potencial que presentan las enzimas producidas en el ciego del cuy, los objetivos del presente estudio fueron cuantificar la actividad de las enzimas hidrolíticas cecales y el efecto de la temperatura y el $\mathrm{pH}$ sobre la actividad de estas enzimas en cuyes (Cavia porcellus).

\section{MATERIAL Y MÉTODOS}

El estudio se llevó a cabo en el Centro de Investigación de Bioquímica y Nutrición de la Facultad de Medicina Humana de la Universidad Nacional Mayor de San Marcos. Se emplearon cinco cuyes machos, mejorados provenientes del Instituto Veterinario de Investigación Tropical y de Altura, localizado a 3320 metros de altitud, en el distrito de El Mantaro, provincia de Jauja, departamento de Junín. Los cuyes fueron seleccionados de una población de 2000 animales, la selección se realizó de manera aleatoria, para lo cual todos los individuos poseían una identificación y utilizando la herramienta de Microsoft Excel para números aleatorios se eligieron los individuos a utilizar. Los cuyes se seleccionaron al final del periodo de engorde (aproximadamente $70 \mathrm{~d}$ de edad) con un peso promedio de $1022 \mathrm{~g}$. Su alimentación fue a base de alfalfa (Medicago sativa) y trébol rojo (Trifolium pratense). Se recolectó el contenido cecal (CC) con una espátula estéril y fue colocado en tubos estériles tipo Falcón de $50 \mathrm{~mL}$ y se almacenaron a $-20^{\circ} \mathrm{C}$, las muestras se procesaron dentro de los $7 \mathrm{~d}$ siguientes. El presente experimento contó con la aprobación del Comité de Ética y Bienestar Animal de la Facultad de Medicina Veterinaria de la Universidad Mayor de San Marcos de Lima Perú.

El CC de cada uno de los cuyes se procesó de manera individual. Se describe brevemente el procesamiento: se colocó $6 \mathrm{~g}$ de CC con $24 \mathrm{~mL}$ de buffer fosfato de sodio (50 mM pH 7) en un vaso de precipitado y se homogeneizó con un agitador magnético refrigerado a $4^{\circ} \mathrm{C}$ por 2 horas. Este homogeneizado se sonicó por cinco tiempos, con una duración de 10 segundos cada uno. Se centrifugó a 10,000 x g por 10 minutos a $4^{\circ} \mathrm{C}$. Los precipitados se lavaron dos veces con buffer fosfato de sodio (50 mM pH 7) y se resuspendieron al 40\% p/v en el mismo buffer. Los restos celulares se removieron por centrifugación $\left(20,000 \times \mathrm{g}\right.$ por 30 minutos a $\left.4^{\circ} \mathrm{C}\right)$. Se tomó el sobrenadante, el cual era un extracto libre de células, se conservaron en crioviales a congelación $\left(-20^{\circ} \mathrm{C}\right)$ para los posteriores ensayos de actividad enzimática. Las actividades de las enzimas cecales (EC) se expresaron como actividad específica ( $\mathrm{U} / \mathrm{mg}$ proteína) y se realizaron por triplicado. Se cuantificó la actividad celulolítica, amilolítica, proteolítica y lipolítica, sometidas a diferentes condiciones de temperaturas $\left(20^{\circ} \mathrm{C}, 30^{\circ} \mathrm{C}\right.$ y $\left.40^{\circ} \mathrm{C}\right)$ у $\mathrm{pH}(5,7$ y 9$)$.

Para medir la actividad amilolítica y celulolítica de las EC, el concentrado enzimático cecal (CEC) fue sometido a un pre-tratamiento. Se colocó en un tubo de ensayo $1 \mathrm{~mL}$ de sustrato, con $0.1 \mathrm{~mL}$ de CEC y $0.4 \mathrm{~mL}$ de soluciones tampón. En el caso de la actividad amilolítica, el sustrato utilizado fue almidón soluble al 1\% en Tris- $\mathrm{HCl}, 50 \mathrm{mM}$, pH 7.5. Para la actividad celulolítica se utilizó el sustrato Carboximetil Celulosa (CMC) a una concentración de $1.5 \mathrm{mg} / \mathrm{mL}$ en fosfato de sodio $0.05 \mathrm{M}$, pH 7. Para medir ambas actividades se incubó tanto el CEC, el sustrato y solución tampón. Se utilizaron 3 soluciones tampón de diferente $\mathrm{pH}$, los cuales fueron: pH 5 (acetato de sodio $50 \mathrm{mM}$ ), pH 7 (fosfato de sodio $50 \mathrm{mM}$ ) y pH 9 (carbonato de sodio $50 \mathrm{mM}$ ). La incubación se realizó por un periodo de dos horas en baño maría a $20^{\circ} \mathrm{C}, 30^{\circ} \mathrm{C}$ y $40^{\circ} \mathrm{C}$. La actividad enzimática se midió con la técnica del 3,5 ácido dinitrosalicilico (DNS), descrito por Miller (1959). La unidad enzimática (U) se definió como la cantidad de glucosa liberado en $\mathrm{g} / \mathrm{L}$ respectivamente.

Para medir la actividad proteolítica de las EC se utilizó la técnica de la azocaseina (Secades\& Guijarro, 1999). La actividad se midió por la aparición del producto, es decir los péptidos coloreados productos de la hidrólisis del sustrato, la azocaseina. La U se definió como la cantidad en la cual se da un incremento en $\mathrm{A}_{420}$ de 0.01 en $30 \mathrm{~min}$, bajo las condiciones del ensayo.

Para medir la actividad lipolítica de las EC se utilizó el método espectrofotométrico descrita por Godoy et. al., 2009. La actividad se midió por la aparición del producto, es decir el cromóforo p-nitrofenol. El sustrato utilizado para esta prueba fue el p-nitrofenilcaprilato, 
el cual fue preparado con acetilnitrilo:DMSO (1:1), la concentración final fue $2.5 \mathrm{mM}$. La U se definió como cantidad de enzima necesaria para formar $1.0 \mathrm{~mol}$ de p-nitrofenol por minuto bajo las condiciones de prueba.

Para cuantificar las proteínas del EC, se utilizó la técnica de Lowryet al. (1951). La proteína patrón fue la albúmina sérica bovina a una concentración de $2.9 \mathrm{~g} / \mathrm{dL}$.

Se utilizó un Diseño Completamente al Azar (DCA) en arreglo factorial $(3 \times 3)$. Los factores a estudiar fueron nivel de temperatura $\left(20,30\right.$ y $\left.40^{\circ} \mathrm{C}\right)$ y nivel $\mathrm{pH}(5,7 \mathrm{y}$ 9) generando 9 tratamientos. El modelo aditivo lineal con arreglo factorial fue el siguiente: $Y i j=\mu+A i+B j+$ ABij+ eijk. Dónde: Yij= observación del i-ésimo nivel de tratamiento en la j-ésima repetición, $\mu=$ media general, $\mathrm{Ai}=$ Efecto del i-mo nivel de temperatura (i: 1, 2,3), Bj = Efecto del j-mo nivel de $\mathrm{pH}(\mathrm{j}: 1,2,3)$, Abij = Efecto de la interacción entre el i-mo nivel de temperatura con el j-mo nivel de $\mathrm{pH}$, eijk = Error experimental. Los datos se analizaron con un ANOVA para un diseño factorial con el paquete estadístico SAS (2002). Se fijó un valor de alfa $<0.05$ para considerar las diferencias entre tratamientos como estadísticamente significativas. Para determinar variaciones entre tratamientos de las medias fueron comparadas mediante la prueba de mínima diferencia significativa aplicando un nivel de significancia de $5 \%$.

\section{RESULTADOS Y DISCUSIÓN}

Las enzimas son productos de las células y por lo tanto pueden obtenerse a partir de tejidos animales, tejidos vegetales o mediante procesos de fermentación empleando microorganismos seleccionados. Los microorganismos representan una excelente fuente de enzimas debido a su amplia diversidad bioquímica y su susceptibilidad a la manipulación genética. En la actualidad, las enzimas más utilizadas en la biotecnología son las hidrolasas, representando aproximadamente el 75\% de las enzimas industriales (Bhat, 2000; Saha, Jordan \& Bothast, 2009). La microbiota del ciego del cuy es una fuente probable de microorganismos que podrían tener potencial biotecnológico. Sin embargo, esta microbiota no ha sido caracterizada. Este es el primer reporte sobre la actividad enzimática en el ciego de cuyes, evidenciando que existe actividad amilolítica, celulolítica, proteolítica y lipolítica. En la Tabla I se presentan los resultados de las cuatro actividades evaluadas en el presente estudio.

Las amilasas hidrolizan moléculas de almidón dando como productos dextrinas y polímeros compuestos progresivamente por unidades de glucosa (Asgher et al., 2007). Se han logrado aislar amilasas a partir de bacterias y hongos presentando características particulares entre ellas. En este estudio, la actividad amilolítica en el ciego de cuyes se incrementó a medida que la temperatura se incrementa. Así, la actividad amilolitica se incrementó en $86 \%$ cuando se aumentó la temperatura de $20^{\circ}$ a $30^{\circ} \mathrm{C}$ y $46 \%$ cuando fue de $30^{\circ} \mathrm{C}$ a $40^{\circ} \mathrm{C}$, siendo similares a los datos reportados en otros estudios (Wind et al., 1994; Hillier et al, 1997). El rango de

Tabla I. Efecto de la temperatura y pH sobre la actividad de las enzimas hidrolíticas del ciego del cuy (Cavia porcellus) (Effect of temperature and pH on the activity of hidrolytic enzymes of the cecal region of the cuy (Cavia porcellus)

\begin{tabular}{|c|c|c|c|c|c|c|}
\hline \multirow{2}{*}{ Tratamientos } & \multirow{2}{*}{ Temperatura $\left({ }^{\circ} \mathrm{C}\right)$} & \multirow{2}{*}{$\mathrm{pH}$} & \multicolumn{4}{|c|}{ ACTIVIDAD ENZIMÁTICA (U/mg proteína) } \\
\hline & & & Amilolítica & Celulolítica & Proteolítica & Lipolítica \\
\hline T1 & 20 & 5 & 0.189 & 0.013 & 0.290 & 3.073 \\
\hline $\mathrm{T} 2$ & 20 & 7 & 0.174 & 0.017 & 0.221 & 2.795 \\
\hline T3 & 20 & 9 & 0.150 & 0.018 & 0.163 & 2.550 \\
\hline T4 & 30 & 5 & 0.330 & 0.015 & 0.183 & 3.014 \\
\hline T5 & 30 & 7 & 0.309 & 0.020 & 0.209 & 2.227 \\
\hline T6 & 30 & 9 & 0.320 & 0.021 & 0.104 & 3.590 \\
\hline $\mathrm{T7}$ & 40 & 5 & 0.441 & 0.021 & 0.140 & 3.175 \\
\hline T8 & 40 & 7 & 0.453 & 0.021 & 0.176 & 2.935 \\
\hline T9 & 40 & 9 & 0.499 & 0.022 & 0.280 & 4.049 \\
\hline \multirow[t]{3}{*}{ Efecto temperatura } & \multicolumn{2}{|r|}{20} & $0.171^{c}$ & $0.016^{c}$ & 0.225 & $2.806^{b}$ \\
\hline & \multicolumn{2}{|r|}{30} & $0.319^{b}$ & $0.019^{b}$ & 0.165 & $2.944^{\mathrm{a}, \mathrm{b}}$ \\
\hline & \multicolumn{2}{|r|}{40} & $0.464^{a}$ & $0.021^{a}$ & 0.199 & $3.386^{\mathrm{a}}$ \\
\hline \multirow[t]{3}{*}{ Efecto $\mathrm{pH}$} & \multicolumn{2}{|r|}{5} & 0.320 & $0.017^{\mathrm{b}}$ & 0.205 & $3.087^{\mathrm{a}, \mathrm{b}}$ \\
\hline & \multicolumn{2}{|r|}{7} & 0.312 & $0.019^{a}$ & 0.202 & $2.653^{\mathrm{b}}$ \\
\hline & \multicolumn{2}{|r|}{9} & 0.323 & $0.020^{\mathrm{a}}$ & 0.183 & $3.396^{\mathrm{a}}$ \\
\hline \multicolumn{3}{|c|}{ FUENTE DE VARIACIÓN } & \multicolumn{4}{|c|}{ PROBABILIDAD } \\
\hline \multicolumn{3}{|l|}{ Temperatura } & $<0.0001$ & $<0.0001$ & 0.2197 & 0.0372 \\
\hline \multicolumn{3}{|l|}{$\mathrm{pH}$} & 0.9085 & $<0.0001$ & 0.7740 & 0.0085 \\
\hline \multicolumn{3}{|l|}{ Temperatura* $\mathrm{pH}$} & 0.6491 & 0.0074 & 0.0166 & 0.0306 \\
\hline
\end{tabular}


temperaturas óptimas para la producción de amilasas bacterianas aisladas de la industrialización de papas o de leche fresca ovina esta entre $35-80^{\circ} \mathrm{C}$ (Wind et al., 1994; ; Konsoula et al., 2004). En cuanto al pH, no afectó la actividad amilolítica, es decir la actividad enzimática fue muy estable en el rango de $\mathrm{pH}$ evaluado, datos que concuerdan con un estudio reciente donde purificaron y caracterizaron bioquímicamente una amilasa de origen bacteriano aislada de fuentes termales (Xiangrong et al., 2017).

Dentro de los microorganismos productores de celulosas existen una diversidad de bacterias y hongos aerobios y anaerobios, mesófilos o termófilos. Estas hidrolasas tienen una amplia variedad de aplicaciones potenciales. La actividad celulolítica en el ciego del cuy se vio afectada tanto por la temperatura como el $\mathrm{pH}$, existiendo interacción entre ambas variables. El efecto de las dos variables sobre la actividad celulolítica es directamente proporcional obteniéndose valores mayores en los niveles más altos evaluados en este estudio. El incremento de la actividad celulolítica fue aproximadamente de $19 \%$ cuando se incrementó la temperatura de 20 a $30^{\circ} \mathrm{C}$ y $11 \%$ cuando el incremento fue de 30 a $40^{\circ} \mathrm{C}$. Los incrementos de la actividad celulolítica cuando se incrementó el pH de 4 a 7 fue de $12 \%$ y tan solo de $5 \%$ cuando se incrementó el pH de 7 a 9, no existiendo diferencia estadística significativa entre estos. Estos datos son similares a los reportados por Gaitán y Pérez (2007) que obtuvieron la mayor actividad enzimática de celulasas de Bacillus sp. y Streptomyces sp., aislados de residuos vegetales y compost, a $\mathrm{pH}$ 7. Además, Howard et al. (2003), mencionaron que el pH óptimo de las celulasas producidas por bacterias abarca un amplio rango, el cual incluye tanto condiciones ácidas como alcalinas, sin embargo, las celulasas producidas por hongos requieren generalmente un $\mathrm{pH}$ ácido. Así, Valiño (1999), menciona que la acción de las enzimas celulasas puede afectarse por numerosos factores, como la adsorción de la enzima al sustrato, la inactivación de la enzima con el tiempo, el pH, la temperatura, la concentración de nitrógeno, además de la inhibición de la enzima con el tiempo de la reacción, entre otros. Respecto al efecto de la temperatura sobre la actividad celulolítica, en este estudio se observó que los valores más altos se dieron a $40^{\circ} \mathrm{C}$, resultados similares a los obtenidos por Gaitán y Pérez (2007), que obtuvieron una actividad más elevada a $37^{\circ} \mathrm{C}$ que a $50^{\circ} \mathrm{C}$.

En el ciego del cuy la actividad amilolítica es casi 10 veces el valor de la actividad celulolítica, similares hallazgos se reportaron en conejos, siendo la actividad celulolítica la menor en todos los segmentos del tracto digestivo, asimismo, reportan que la actividad amilolítica en el ciego es casi 6 veces la actividad celulolítica. (Marounek, Vovk\&Skrivanová, 1995). Estos hallazgos son consistentes con la baja digestibilidad de la fibra cruda en conejos, la cual ha sido reportada por diversos autores. Slade y Hintz (1982) demostraron que el cuy es tan eficiente como el caballo y pony en la digestión de la fibra cruda. Estudios realizados en otras especies herbívoras y omnívoros evidenciaron alta actividad amilolítica es utilizada en la descomposición del polisacárido en la dieta (Hidalgo, Urea \& Sanz, 1999.; Ademuloet al., 2017), debido a eso los valores que se reportan son más elevados que la actividad celulolítica. Los datos respecto a la baja actividad de la celulasa en el ciego del cuy podrían sugerir que existen otras enzimas que contribuirían a la hidrólisis de la fibra vegetal o que no es tan eficiente en la utilización del forraje.

Las EC de cuyes no mostraron una actividad proteolítica uniforme, tuvieron respuestas diferentes para cada condición a la que estuvo expuesta, sin embargo, estos datos no difirieron entre ellos. Estudios en proteasas comerciales (Novo Nordisk $\AA$ ) sugieren que la mayor actividad será a $\mathrm{pH}$ alcalino $(8,5-10,5)$ y a la temperatura de $55^{\circ} \mathrm{C}$. (Cegarra, Gacén \& Cayuela 2000). García (1996), evaluó las proteasas bacterianas en sistemas de depuración biológica; encontrando que la máxima actividad fue a $\mathrm{pH} 7$, por debajo o encima de este valor la actividad proteolítica disminuye, siendo mayor cuando el pH es ácido. También reporta la mayor actividad en el rango de temperatura que va desde $10^{\circ} \mathrm{C}-35^{\circ} \mathrm{C}$.

La actividad lipolítica se vio afectada por la temperatura y el $\mathrm{pH}$ existiendo interacción entre ambas variables. El efecto de la temperatura sobre la actividad lipolítica fue directamente proporcional obteniéndose el valor más alto $40^{\circ} \mathrm{C}$. El incremento de la actividad celulolítica fue aproximadamente de $4 \%$ cuando se incrementó la temperatura de 20 a $30^{\circ} \mathrm{C}$ y $15 \%$ cuando el incremento fue de 30 a $40^{\circ} \mathrm{C}$. El efecto del $\mathrm{pH}$ no fue directamente proporcional ya que cuando se incrementó el pH de 4 a 7 la actividad celulolítica disminuyó en $14 \%$ y se incrementó en $28 \%$ cuando se incrementó el pH de 7 a 9. Coca et al. (2001) evaluó la actividad de lipasas de los hongos Aspergillus niger y A. fumigatus, aislados de caña de azúcar,manteniendo el $\mathrm{pH}$ estable a 7 , obteniendo la máxima actividad enzimática en un rango de 40 y $60^{\circ} \mathrm{C}$ para $A$. niger y $80^{\circ} \mathrm{C}$ para $A$. fumigatus, luego de estas temperaturas la actividad enzimática cayó, atribuidas a una inactivación térmica. En el presente estudio no se evidencio una inactivación térmica, ya que no se determinó la actividad máxima, pues no se evaluó temperaturas extremas. Al igual que los resultados presentados por Coca et al. (2001) las tendencias de la actividad enzimática de las lipasas tienden a incrementarse con el efecto de la elevación de la temperatura. En el estudio realizado por Castro et al. (2003), evaluó la lipasa de Bacillusthermoleovorans, la lipasa CCR11, aislado de aguas termales, tiene un $\mathrm{pH}$ óptimo de 9 y es estable en un rango de $\mathrm{pH}$ de $5 \mathrm{a}$ 11. La temperatura óptima de esta lipasa fue de $60^{\circ} \mathrm{C}$.

\section{CONCLUSIONES}

Se logró cuantificar la actividad de las enzimas hidrolíticas cecales del cuy. Existe interacción entre temperatura y $\mathrm{pH}$ para la actividad celulolítica, lipolítica y proteolítica. La actividad amilolítica solo es afectada por la temperatura, teniendo mayor actividad enzimática a mayor temperatura $\left(40^{\circ} \mathrm{C}\right)$. La actividad amilolítica es estable a cualquiera de los tres $\mathrm{pH}$ evaluados. La actividad amilolítica fue 10 veces mayor que la celulolítica.

\section{BIBLIOGRAFÍA}


Ademolu, KO, Ojo, VO, Bamidele, JA, Adelabu, AB I, Ebenso, I \&ldowu, AB 2017 "Feeding pattern and gut enzymes activity of Giant African land snail (Archachatinamarginata) during growth phases" Archivos de ZOotecnia 66 (253): 29-34

Asgher M, JavaidAsad, M, Rahman SU \&Legge RL 2007, "A thermostable a-amylase from a moderately thermophilicBacillus subtilis strain for starch processing. Journal of food Engineering. Volume 79, Issue 3, Pages 950-955

Bhat, MK 2000, "Cellulases and related enzymes in biotechnology" Biotechnology Advances, 18:355-383

Buchholz, K, Kasche, V \&Bornscheuer, UT 2012, Biocatalysts and Enzyme Technology, ed. John Wiley\&Sons, 201,. 2da edition, 626 p.

Castro, DL, Rodriguez, C, Valerio, G \&Oliart, RM 2003, "Purificación y caracterización parcial de la lipasa producida por la bacteria termófila BacillusthermoleovoransCCR 1 1", trabajo presentado en el X Congreso Nacional de Biotecnología y Bioingeniería, Puerto Vallarta, Jalisco- México, 8 a 12 de septiembre.

Cegarra, J, Gacén, J \& Cayuela, D 2000, "Blanqueo de lana en presencia de proteasas", Boletin Intexter U.P.C. N N 118: 41-43.

Coca, J, Hernández, O, Berrio, R, Martínez, S, Díaz, E, \&Dustet, JC 2001, "Producción y caracterización de lipasas de Aspergillus nigery A. fumigatus", Biotecnología aplicada 18: 216- 220

Esquerre, J, Valenzuela, A \& Candela, E 1974, “Digestión microbiana en cuyes criollos de la altura", Rev. Inv. Pec. (IVITA), Universidad Nacional Mayor de San Marcos 3 (19: 67-76).

Food and AgricultureOrganization of theUnitedNations 1997, Producción de cuyes (Cavia porcellus), Roma,visto 15 de julio del 2017, <http:// www.fao.org/docrep/W6562S/W6562S00.htm/>

Gaitán, D \& Pérez, L 2007, "Aislamiento y evaluación de microorganismos celulolíticos a partir de residuos vegetales frescos y en compost generados en un cultivo de crisantemo (Dendhranthema grandiflora)", Tesis para optar el título de Microbióloga Industrial, Bogotá: Pontificia Universidad Javeriana, $97 \mathrm{p}$.

García, J 1996, "Actividad de las proteasas bacterianas en sistemas de depuración biológicos", Tesis para optar el grado de Doctor en Ciencias Químicas, Madrid, España, Universidad Complutense de Madrid. 252 pp.

Godoy, MG, Gutarra, MLE, Maciel, FM, Felix, SP, Bevilaqua, JB, Machado, OLT, \&Freire DMG 2009, "Use of a low-cost methodology for biodetoxification of castor bean waste and lipase production", Enzyme and Microbial Technology, 44, 317-322.

Gomez, BC \& Vergara, V 1993, Fundamentos de Nutrición y Alimentación, I Curso Nacional de Capacitación en Crianzas Familiares, PG 30-50 INIA - EELM - EEBI.

Hidalgo MC. Urea, E and Sanz, A 1999, "Comparative study of digestive enzymes in fish with different nutritional habits. Proteolytic and amylase activities", Aquaculture 170: 267-283

Hillier, P, Wase, DAJ, Emery, AN \& Solomons, GL 1997, "Instability of a-amylase production and morphological variation incontinuous culture of Bacillus amyloliquefaciens is associated with plasmid loss", Process Biochemistry, 32, 51-59.

Hintz, HF, 1961, "The effect of autoclaving on the nutritive value of bay", Thesis. Cornell University, Ithaca, New York.
Howard, R, Abotsi, E, Rensburg, J \& Howard, S 2003, "Lignocellulose Biotechnology: issues bioconversion and enzyme production", African Journal of Biotechnology. 2: 602-619.

Konsoula, Z \&Liakopoulou-Kyriakides, M 2004, "Thermostable, aamylase production by Bacillus subtilis entrapped in calcium alginate gel capsules", Enzyme and Microbial Technology. 2004, 39, 690-696.

Lindal, J 2004, Enzimas: Clasificación, cinética y control. En: Devlin T, ed. Bioquímica: libro de texto con aplicaciones clínicas. 4ta edición, España. Editorial Reverté S.A. p 413-463.

Lowry, OH, Rosebrough, NJ, Farr, AL \& Randall, RJ 1951,"Protein measurement with the Folin phenol reagent", Journal of Biological Chemistry 193: 265-275.

Marounek, M, Vovk, SJ, \&Skrivanová, V 1995, "Distribution of activity of hydrolytic enzymes in the digestive tract of rabbits" British Journal of Nutrition 73, 463-469.

Miller, GL 1959, "Use of dinitrosalicylic acid reagent for determination of reducing sugar", Analytical Chemistry 31 (3): 426-428.

Saha, BC, Jordan, DB \&Bothast, RJ 2009, Enzymes, industrial (overview), Encyclopedia of Microbiology, Academic Press, Oxford.

SAS 2002, SAS User's Guide: Statistics, Version 9.0. SAS Instlnc,Cary, NC, USA.

Secades, P \&Guijarro, JA 1999, "Characterization and purification of an extracellular protease from the fish pathogen Yersinia rukeri and effect of culture conditions on production" Applied and Environmental Microbiology. 65(9):3969-3975.

Slande, LM\&Hintz, HF 1982, "Comparasion of digestion in horses, ponies, rabbits and guinea pigs" British Journal of Nutrition 58: 149-158.

Valiño, E 1999, "Fermentación en estado sólido del bagazo de caña por especies de hongos conidiales productores de celulasas" Tesis PhD. Instituto de Ciencia Animal. La Habana, Cuba

Wind, RD, Buitelaar, RM, Eggink, G, Huizing, HJ \&Dijkhuizen, L 1994, "Characterization of a new Bacillus stearothermophilus a highly thermostable a-amylase-producing strain", Applied Microbiology and Biotechnology 1994, 41, 155-162.

Xiangrong, W, Yuxia, W, Bending, T, Xianghua, C \&Jianhua, C 2018, "Purification and biochemical characterization of a thermostable and acid-stable alpha-amylase from Bacillus licheniformis B4-423" International Journal of Biological Macromolecules. 2017 Dec 9; 109:329-337. doi: 10.1016/j.ijbiomac.2017.12.004 